\title{
MULTIPLE ANTIBIOTIC RESISTANCE OF AIRBORNE BACTERIA IN OUTDOOR MARKETS IN ADO-EKITI METROPOLIS
}

Aladejana, O. M. ${ }^{1}$, Oluyege, J. O. ${ }^{2}$, Olowomofe, T. O. ${ }^{2}$ Obayemi, I. E ${ }^{2}$ and Oluyege, D.E 3

${ }^{1}$ Department of Microbiology, Faculty of Sciences, Odeomu Osun State, Nigeria

${ }^{2}$ Department of Microbiology, Faculty of Sciences, Ekiti State University, Ado - Ekiti, Nigeria

${ }^{3}$ Institute of Food Security, Environmental Resources and Agricultural Research, Federal University of Agriculture, Abeokuta, Ogun State, Nigeria

\section{REZISTENTNOST BAKTERIJA U VAZDUHU NA VIŠESTRUKE ANTIBIOTIIKE NA OTVORENIM TRŽNICAMA U METROPOLI ADO-EKITI

\author{
Aladejana, O. M. ${ }^{1}$, Oluyege, J. O. ${ }^{2}$, Olowomofe, T. O. ${ }^{2}$ Obayemi, I. E2 i Oluyege, D.E ${ }^{3}$ \\ ${ }^{1}$ Katedra za mikrobiologiju, Prirodno-matematički fakultet, država Odeomu Osun, Nigerija \\ ${ }^{2}$ Katedra za mikrobiologiju, Prirodno-matematički fakultet, Državni univerzitet Ekiti, Ado-Ekiti, Nigerija \\ ${ }^{3}$ Institut za bezbednost hrane, resurse životne sredine i poljoprivredno istraživanje, \\ Savezni univerzitet za poljoprivredu, Abeokuta, država Ogun, Nigerija
}

\begin{abstract}
The emergence of multiple antibiotic resistant strains in the environment, particularly in a densely populated market is a problem to the infection management. The prevalence of antibiotic-resistant airborne bacteria was examined in five different outdoor markets in Ado Ekiti, Ekiti State, Nigeria. The sample collection was taken between 3-5pm when activities at the market place were at the peak. The bacteria isolates were identified and their antibiotic susceptibility to fourteen different antibiotics was carried out. Six genera of bacteria were recovered, Staphylococcus (16.7\%), Escherichia (18.6\%), Pseudomonas (21.6\%), Enterococcus (19.6\%), Salmonella (10.8\%) and Klebsiella (12.7\%). The airborne isolates showed the highest resistance to Pefloxacin (52.3\%), followed by Augumentin (44.7\%). The lowest resistance was to Erythromycin (5.8\%). All of Salmonellae typhimurium, and Escherichia coli were multiple antibiotic resistant (MAR), while a great percentage of otherisolates were also MAR (Staphylococcus aureus (88.3\%), Pseudomonas aeruginosa (90.9\%), Klebsiella pneumoniae (92.3\%), and Enterococcus spp (85\%). These results show that the air in the outdoor markets studied was highly contaminated with a great diversity of MAR bacteria. The findings of this study will be helpful in the intervention of health officials and policy makers in decision making towards the effective control of infections caused by the airborne bacteria in the outdoor markets.
\end{abstract}

Keywords: Antibiotic resistance, Open market, airborne bacteria, Public health, Infections.

\section{SAŽETAK}

Pojava rezistetnih sojeva bakterija na višestruke antibiotike $u$ životnoj sredini posebno u gusto naseljenoj tržnici je problem $u$ tretiranju infekcije. Rasprostranjenost bakterija u vazduhu, rezistetnih na antibiotike je bilo ispitivano u pet različitih otvorenih tržnica u Ado Ekiti, država Ekiti, Nigerija. Skupljanje uzoraka je vršeno između 15 i 17 časova kada su aktivnosti na tržnicama bile na vrhuncu. Bakterijski izolati su bili identifikovani i njihova antibiotska susceptibilnost na 14 razlicitih antibiotika je bila izvedena. Šest sojeva bakterija se oporavilo Staphylococcus (16.7\%), Escherichia (18.6\%), Pseudomonas (21.6\%), Enterococcus (19.6\%), Salmonella (10.8\%) and Klebsiella (12.7\%). Izolati iz vazduha su pokazali najveću rezistentnost na Pefloxacin (52.3\%) i Augumentin (44.7\%). Najniža rezistentnost je bila na Erythromycin (5.8\%). Sve Salmonellae typhimurium, i Escherichia coli su bile rezistentne na višestruke antibiotike dok je veliki procenat drugih izolata takođe bio rezistentan na višestruke antibiotike Staphylococcus aureus (88.3\%), Pseudomonas aeruginosa (90.9\%), Klebsiella pneumoniae (92.3\%), i Enterococcus spp (85\%). Ovi rezultati pokazuju da je vazduh na otvorenim tržnicama koje su proučavane bio veoma kontaminiran velikim spektrom bakterija koje su rezistentne na višestruke antibiotike. Nalazi ove studije će biti od pomoći zdravstvenim službenicima $i$ političarima da donose odluke u cilju efikasne kontrole infekcija prouzrokovane bakterijama u vazduhu na otvorenim tržnicama.

Ključne reči: rezistentnost na antibiotike, otvorena tržnica, bakterije u vazduhu, javno zdravlje, infekcije. 


\section{BACKGROUND}

Although there are significantly fewer atmospheric microorganisms than there are in the oceans and in the soil, there is still a large enough number of bacteria, fungi, viruses, toxins, endotoxins, pollens, metabolites, or any other biological materials that can affect the atmosphere (1). In urban areas, the vehicular intensity adds to dust suspension with the subsequent addition of bioaerosols which varies greatly at different sites due to different point sources. The meteorological parameters like wind direction, wind speed, temperature, and relative humidity determine the suspension, transportation, and deposition of airborne microbes (2). Once suspended in the air column, microoganisms have the opportunity to travel long distances with the help of wind and precipitation, increasing the occurrence of a widespread disease by these microorganisms. These aerosols are ecologically significant in many environments including hospitals, animal sheds, clean-rooms, pharmaceutical facilities and spacecraft environments because they can be associated with the disease in humans, animals and plants therefore making the efficient monitoring crucial. In the outdoor market where different farm produce are brought for sales from both far and nearby farms have an increased diversity of microorganisms. Also people come from far and near to buy things and their activities such as walking, sneezing and talking will also lead to the introduction of varied microorganisms. The emergence of multiple antibiotic resistant strains in this type of environment is a great concern. The market provides a potentially favourable environment for the proliferation and transport of antibiotic-resistant airborne microbes.

The aim of this study was to determine the public health risk factor in terms of the incidence of multiple antibiotic resistant bacteria in the air environment of some selected open markets in Ado-Ekiti, Nigeria.

\section{MATERIALS AND METHODS}

\section{Study Location}

The study site are four outdoor markets all in Ado- Ekiti Ekiti State Nigeria. The outdoor markets examined were Okesa, Oba-palace, Odo-Ado and Mojere. (Plate 1).

\section{Isolation and Identification of Bacteria Cells From Outdoor Environment}

The isolates were obtained in the afternoon (3- $5 \mathrm{pm}$ ) which had a high number of growth. The bacteria cells were isolated by the use of settling plate method or sedimentation method of Pasquarella et al 2001 (3), using the appropriate media and incubated at $37 \mathrm{oC}$ for $24 \mathrm{hrs}$. Identification and characterization of bacteria isolates were carried out using the cultural, morphological and biochemical test according to BERGEY's Manual of Systematic Bacteriology.

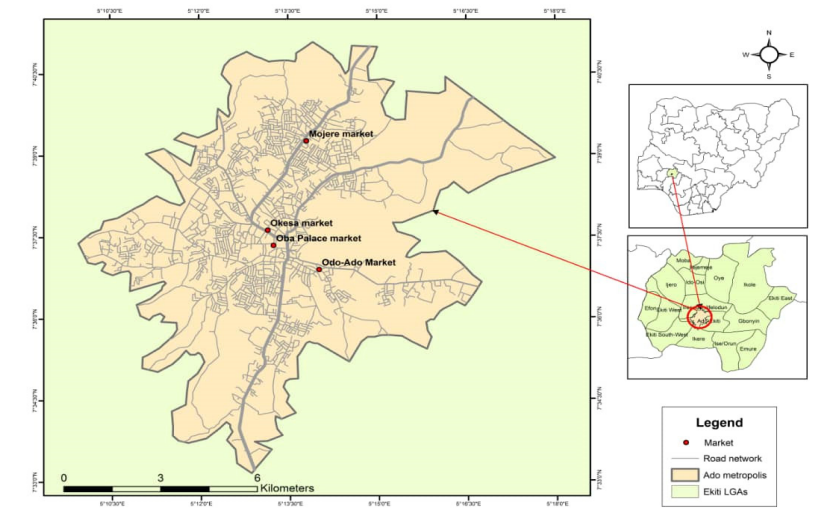

Plate 1. Map of the Study Area in Ado- Ekiti Metropolis

\section{Determination of Antimicrobial Activities}

The antibiotic susceptibility tests were performed to determine the phenotypic resistance patterns of the identified bacterial isolates to commonly used antibiotics in Nigeria. This test was carried out by Kirby-Bauer's disc diffusion method on Mueller-Hinton agar. The antibiotics screened against the bacterial isolates were pefloxacin, tarivid, septrin, gentamycin, amoxacillin, ciprofloxacin, ampiclox, chloranphenicol, zinnacef, sparfloxacin, rocephin, augumentin, erythromycin, streptomycin. The inoculum was standardized to 0.5 Macfarlane standard, Plates were inoculated and then incubated at $37 \mathrm{oC}$ for 24 hours. The diameter zones of inhibition were measured and interpreted according to the Clinical Laboratory Standard Institute guidelines.

The MAR index for each of the airborne bacterial isolates was calculated as indicated below: a/bc where a represents the aggregate antibiotic resistance score of all the isolates from the samples and $\mathrm{c}$ is the number of the isolates from the samples (4)

\section{RESULTS}

\section{Bacterial Population of Outdoor Air}

The colonial growth of bacteria isolates from the outdoor Market air on Nutrient Agar (NA) ranged from 82 to 201, Mannitol Salt Agar (MSA) from 37 to 69, MacConkey (MAC) Agar from 21 to 32, Salmonella-Shigella (SS) agar from 5 to 16 and Eosin Methylene Blue (EMB) agar ranged from 0 to 3 (Table 1). 
Table 1. Growth of Microorganisms on Agar Plates Exposed in Outdoor Market Environment, Ado-Ekiti, Ekiti State.

\begin{tabular}{lllllll}
\hline $\begin{array}{l}\text { LOCATION } \\
\text { Time (3-5pm) }\end{array}$ & NA & MSA & MCA & SS & EMBA & Temperature \\
& & & & & & \\
\hline Location 1 & $210.0 \pm 3.0$ & $69.0 \pm 1.732$ & $52.0 \pm 0$ & $16.0 \pm 1.0$ & $3.0 \pm 0.0$ & $29 \pm 1^{\circ} \mathrm{C}$ \\
Location 2 & $188.0 \pm 2.0$ & $50.0 \pm 5.0$ & $47.0 \pm 5.568$ & $12.0 \pm 1732.0$ & $1.0 \pm 0.0$ & $28 \pm 1^{\circ} \mathrm{C}$ \\
Location 3 & $157.0 \pm 2.0$ & $43.0 \pm 1.0$ & $32.0 \pm 2.0$ & $7.0 \pm 2.0$ & $2.0 \pm 1.0$ & $29 \pm 1^{\circ} \mathrm{C}$ \\
Location 4 & $84.0 \pm 4.0$ & $37.0 \pm 3.0$ & $21.0 \pm 1.0$ & $5.0 \pm 1.0$ & $0.0 \pm 0$ & $28 \pm 1^{\circ} \mathrm{C}$ \\
\hline
\end{tabular}

Results are presented as mean \pm standard error mean (SEM)

Location 1 = Oba market, Location 2 = Mojere market, Location 3 =Okesa market, Location $4=$ Odo-Ado Market, MSA

$=$ Manitol Salt Agar, NA = Nutrient Agar, EMB = Eosin Methylene Blue agar, MAC = MacConkey agar, SS = Salmonella-Shigella agar

\section{Identification of Bacteria Isolates from Outdoor Market Environment in Ado Ekiti, Ekiti State.}

Based on their morphological characteristics and biochemical tests, six genera of bacteria were isolated which are Staphylococcus (16.7\%), Escherichia (18.6\%), Pseudomonas (21.6\%), Enterococcus (19.6\%), Salmonella (10.8\%) and Klebsiella $(12.7 \%)$ as shown in Table 2 .

Table 2. Distribution of Bacteria Isolates from Outdoor Air

\begin{tabular}{lll}
\hline Bacteria Species & Bacterial number & Percentage Distribution (\%) \\
\hline Staphylococcus aureus & 17 & 16.7 \\
Salmonella typhimurium & 11 & 10.8 \\
Escherichia coli & 19 & 18.6 \\
Pseudomonas aeruginosa & 22 & 21.6 \\
Klebsiella pneumonia & 13 & 12.7 \\
Enterococcus spp & 20 & 19.6 \\
\hline Total & $\mathbf{1 0 2}$ & $\mathbf{1 0 0}$ \\
\hline
\end{tabular}

\section{Antibiotic Resistance of Bacteria Isolates from Outdoor Market in Ado Ekiti, Ekiti State}

The antibiotic susceptibility test was carried out on the bacteria isolates: $S$. aureus (17), S. typhimurium (11), E. coli (19), P. aeruginosa (22), K. pneumoniae (13), and Enterococcus (20). Table 3 shows the antibiotic resistance pattern of the bacteria isolated from the air where $S$. aureus had the highest resistance percentage $(53 \%)$ to pefloxacin and the lowest resistance percentage (18\%) to streptomycin and rocephin with no resistance to erythromycin (0\%). S. typhimurium had the highest resistance percentage $(100 \%)$ to Amoxacillin and the lowest resistance percentage (18\%) to Streptomycin, Tarivid and Chloramphenicol with no resistance to Septrin, Ciprofloxacin and Sparfloxacin. E. coli exhibited the highest resistance $(100 \%)$ to Gentamycin and the lowest resistance $(5 \%)$ to Chloramphenicol with no resistance to Oflaxacin $(0 \%), P$. aeruginosa demonstrated the highest percentage resistance $(100 \%)$ to Augmentin and the lowest resistance $(5 \%)$ to Gentamycin with no resistance to Pefloxacin, K. pneumonia exhibited the highest resistance $(92 \%)$ to Pefloxacin and the lowest resistance (23\%) to Streptomycin with no resistance to Chloramphenicol and Septrin, Enterococcus spp demonstrated the highest resistance (60\%) to Ciprofloxacin and the lowest resistance (5\%) to Gentamycin with no resistance to Amoxacillin with the lowest percentage resistance $(0 \%)$ 
Table 3. Antibiotic Resistant Pattern of Bacteria Isolated from Air

\begin{tabular}{|c|c|c|c|c|c|c|c|c|c|}
\hline \multirow{2}{*}{$\begin{array}{l}\mathbf{S} / \\
\mathbf{N}\end{array}$} & \multirow[t]{2}{*}{ Antibiotics } & \multirow[t]{2}{*}{ Code } & \multicolumn{6}{|c|}{ Percentage resistance $\%$} & \multirow{2}{*}{$\begin{array}{c}\text { Overall Per- } \\
\text { centage }(\%) \\
\text { resistance on } \\
\text { bacteria isolate }\end{array}$} \\
\hline & & & $\begin{array}{l}\text { S. aureus } \\
\mathrm{n}=17\end{array}$ & $\begin{array}{l}\text { S. typhi- } \\
\text { murium } \\
\mathrm{n}=11\end{array}$ & $\begin{array}{l}\text { E. coli } \\
\mathrm{n}=19\end{array}$ & $\begin{array}{c}P . \text { aeru- } \\
\text { ginosa } \\
\mathrm{n}=22\end{array}$ & $\begin{array}{c}K . \\
\text { pneu- } \\
\text { moniae } \\
\mathrm{n}=13\end{array}$ & $\begin{array}{c}\text { Entero- } \\
\text { coccus } \\
\text { spp } \\
\mathrm{n}=20\end{array}$ & \\
\hline 1 & Pefloxacin & PEF & 53 & 73 & 53 & 0 & 92 & 45 & 52.7 \\
\hline 2 & Tarivid & OFX & - & 18 & 0 & 27 & 54 & - & 16.5 \\
\hline 3 & Septrin & SXT & 47 & 0 & 11 & 41 & 0 & 40 & 23.2 \\
\hline 4 & Gentamycin & $\mathrm{CN}$ & 29 & 8 & 100 & 5 & 62 & 5 & 34.8 \\
\hline 5 & Amoxacillin & $\mathrm{AM}$ & 29 & 100 & 63 & 55 & 46 & 5 & 49.7 \\
\hline 6 & Ciprofloxacin & CPX & 47 & 0 & 68 & 23 & 38 & 60 & 39.3 \\
\hline 7 & Ampiclox & APX & 41 & - & - & - & - & 55 & 19 \\
\hline 8 & Chloranphenicol & $\mathrm{CH}$ & - & 18 & 5 & 32 & 0 & - & 9.2 \\
\hline 9 & Zinnacef & Z & 35 & - & - & - & - & 25 & 10 \\
\hline 10 & Sparfloxacin & SP & - & 0 & 58 & 27 & 46 & - & 21.8 \\
\hline 11 & Rocephin & $\mathrm{R}$ & 18 & - & - & - & - & 45 & 10.5 \\
\hline 12 & Augumentin & $\mathrm{AU}$ & - & 64 & 58 & 100 & 46 & - & 44.7 \\
\hline 13 & Erythromycin & $\mathrm{E}$ & - & - & - & - & - & 35 & 5.8 \\
\hline 14 & Streptomycin & $\mathrm{S}$ & 18 & 18 & 21 & 23 & 23 & 25 & 21.3 \\
\hline
\end{tabular}

Table 3. Multiple Antibiotic Resistance Pattern of Staphylococcus aureus from Outdoor Market Air in Ado Ekiti, Ekiti State

\begin{tabular}{|c|c|c|c|c|c|}
\hline $\mathbf{S} / \mathbf{N}$ & $\begin{array}{l}\text { No of } \\
\text { Antibi- } \\
\text { otic }\end{array}$ & $\begin{array}{l}\text { Fre- } \\
\text { quency }\end{array}$ & $\begin{array}{l}\% \\
\text { MAR } \\
n=17\end{array}$ & $\begin{array}{l}\text { Antibiotic resistance } \\
\text { patterns }\end{array}$ & MAR Index \\
\hline 1 & 2 & 2 & 11.8 & $\begin{array}{l}\text { CPX } \\
\mathrm{Z}\end{array}$ & 0.008 \\
\hline 2 & 4 & 2 & 11.8 & $\begin{array}{l}\mathrm{APX} / \mathrm{SXT} \\
\mathrm{PEF} / \mathrm{CPX}\end{array}$ & 0.017 \\
\hline 3 & 7 & 6 & 41.2 & $\begin{array}{l}\mathrm{PEF} / \mathrm{CN} / \mathrm{AM} \\
\mathrm{PEF} / \mathrm{APX} / \mathrm{CPX} \\
\mathrm{APX} / \mathrm{AM} / \mathrm{SXT} \\
\mathrm{CN} / \mathrm{Z} / \mathrm{SXT} \\
\mathrm{PEF} / \mathrm{APX} / \mathrm{Z} \\
\mathrm{PEF} / \mathrm{APX} / \mathrm{CPX}\end{array}$ & 0.037 \\
\hline 4 & 8 & 5 & 29.4 & $\begin{array}{l}\mathrm{PEF} / \mathrm{Z} / \mathrm{R} / \mathrm{SXT} \\
\mathrm{PEF} / \mathrm{CN} / \mathrm{APX} / \mathrm{AM} \\
\mathrm{PEF} / \mathrm{Z} / \mathrm{R} / \mathrm{SXT} \\
\mathrm{PEF} / \mathrm{APX} / \mathrm{Z} / \mathrm{CPX} \\
\mathrm{R} / \mathrm{CPX} / \mathrm{S} / \mathrm{SXT}\end{array}$ & 0.034 \\
\hline 5 & 5 & 2 & 11.8 & $\mathrm{CN} / \mathrm{AM} / \mathrm{CPX} / \mathrm{S} / \mathrm{SXT}$ & 0.021 \\
\hline
\end{tabular}

Pefloxacin=PEF, Tarivid=OFX, Septrin=SXT, Gentamycin=CN, Amoxacillin=AM, Ciprofloxacin $=\mathrm{CPX}$, Ampiclox $=\mathrm{APX}$, Chloramphenicol $=\mathrm{CH}$, Zinnacef $=\mathrm{Z}$, Sparfloxa$\operatorname{cin}=\mathrm{Z}$, Rocephin $=\mathrm{R}$, Augumentin $=\mathrm{AU}$, Erythromycin $=\mathrm{E}$, Streptomycin $=\mathrm{S}$. 
Table 4. Antibiotic Resistance Pattern of Salmonella typhimurium from Outdoor Market Air in Ado Ekiti, Ekiti State

\begin{tabular}{|c|c|c|c|c|c|}
\hline $\mathbf{S} / \mathbf{N}$ & $\begin{array}{l}\text { No of An- } \\
\text { tibiotic }\end{array}$ & $\begin{array}{l}\text { Fre- } \\
\text { quency }\end{array}$ & $\begin{array}{l}\% \\
\text { MAR } \\
n=11\end{array}$ & $\begin{array}{l}\text { Antibiotic re- } \\
\text { sistance patterns }\end{array}$ & MRA Index \\
\hline 1 & 7 & 4 & 36.4 & $\begin{array}{l}\mathrm{AM} / \mathrm{AU} / \mathrm{CN} \\
\mathrm{PEF} / \mathrm{CH} / \mathrm{AM} \\
\mathrm{CH} / \mathrm{AM} / \mathrm{CN} \\
\mathrm{PEF} / \mathrm{AM} / \mathrm{AU}\end{array}$ & 0.046 \\
\hline 2 & 7 & 7 & 63.6 & $\begin{array}{l}\mathrm{PEF} / \mathrm{AM} / \mathrm{AU} / \mathrm{CN} \\
\mathrm{OFX} / \mathrm{S} / \mathrm{AM} / \mathrm{CN} \\
\mathrm{PEF} / \mathrm{S} / \mathrm{AM} / \mathrm{CN} \\
\mathrm{PEF} / \mathrm{AM} / \mathrm{AU} / \mathrm{CN} \\
\mathrm{PEF} / \mathrm{AM} / \mathrm{AU} / \mathrm{CN} \\
\mathrm{PEF} / \mathrm{OFX} / \mathrm{AM} / \mathrm{AU} \\
\mathrm{PEF} / \mathrm{AM} / \mathrm{AU} / \mathrm{CN}\end{array}$ & 0.046 \\
\hline
\end{tabular}

Table 6. Antibiotic Resistance Pattern of Escherichia coli from Outdoor Market Air in Ado Ekiti, Ekiti State

\begin{tabular}{|c|c|c|c|c|c|}
\hline $\mathbf{S} / \mathbf{N}$ & $\begin{array}{l}\text { No of Anti- } \\
\text { biotic }\end{array}$ & Frequency & $\begin{array}{l}\% \text { MAR } \\
n=19\end{array}$ & Antibiotic resistance patterns & MAR Index \\
\hline 1 & 2 & 1 & 5.3 & $\mathrm{AM} / \mathrm{CN}$ & 0.008 \\
\hline 2 & 3 & 1 & 5.3 & $\mathrm{SP} / \mathrm{AU} / \mathrm{CN}$ & 0.011 \\
\hline 3 & 9 & 8 & 42.1 & $\begin{array}{l}\mathrm{SP} / \mathrm{CPX} / \mathrm{AM} / \mathrm{CN} \\
\mathrm{PEF} / \mathrm{S} / \mathrm{CPX} / \mathrm{CN} \\
\mathrm{PEF} / \mathrm{CPX} / \mathrm{AU} / \mathrm{CN} \\
\mathrm{CPX} / \mathrm{AM} / \mathrm{AU} / \mathrm{CN} \\
\mathrm{SP} / \mathrm{CPX} / \mathrm{AM} / \mathrm{CN} \\
\mathrm{S} / \mathrm{SXT} / \mathrm{CH} / \mathrm{CN} \\
\mathrm{PEF} / \mathrm{CPX} / \mathrm{AM} / \mathrm{CN} \\
\mathrm{PEF} / \mathrm{SP} / \mathrm{AM} / \mathrm{CN}\end{array}$ & 0.034 \\
\hline 4 & 9 & 8 & 42.1 & $\begin{array}{l}\mathrm{SP} / \mathrm{CPX} / \mathrm{AM} / \mathrm{AU} / \mathrm{CN} \\
\mathrm{PEF} / \mathrm{S} / \mathrm{SXT} / \mathrm{AU} / \mathrm{CN} \\
\mathrm{PEF} / \mathrm{S} / \mathrm{SP} / \mathrm{CPX} / \mathrm{CN} \\
\mathrm{SP} / \mathrm{CPX} / \mathrm{AM} / \mathrm{AU} / \mathrm{CN} \\
\mathrm{PEF} / \mathrm{CPX} / \mathrm{AM} / \mathrm{AU} / \mathrm{CN} \\
\mathrm{SP} / \mathrm{CPX} / \mathrm{AM} / \mathrm{AU} / \mathrm{CN} \\
\mathrm{PEF} / \mathrm{SP} / \mathrm{CPX} / \mathrm{AU} / \mathrm{CN} \\
\mathrm{PEF} / \mathrm{SP} / \mathrm{AM} / \mathrm{AU} / \mathrm{CN}\end{array}$ & 0.034 \\
\hline 5 & 6 & 1 & 5.3 & $\mathrm{PEF} / \mathrm{SP} / \mathrm{CPX} / \mathrm{AM} / \mathrm{AU} / \mathrm{CN}$ & 0.023 \\
\hline
\end{tabular}

Pefloxacin $=$ PEF, Tarivid $=$ OFX, Septrin $=\mathrm{SXT}$, Gentamycin $=\mathrm{CN}$, Amoxacillin $=\mathrm{AM}$, Ciprofloxacin $=\mathrm{CPX}$, Ampiclox $=\mathrm{APX}$, Chloramphenicol $=\mathrm{CH}$, Zinnacef $=\mathrm{Z}$, Sparfloxacin $=\mathrm{Z}$, Rocephin $=\mathrm{R}$, Augumentin $=\mathrm{AU}$, Erythromycin $=\mathrm{E}$, Streptomycin $=\mathrm{S}$. 
Table 7. Antibiotic Resistance Pattern of Pseudomonas aeruginosa from Outdoor Market Air in Ado Ekiti, Ekiti State

\begin{tabular}{|c|c|c|c|c|c|}
\hline $\mathbf{S} / \mathbf{N}$ & $\begin{array}{l}\text { No of } \\
\text { Antibi- } \\
\text { otic }\end{array}$ & $\begin{array}{l}\text { Fre- } \\
\text { quency }\end{array}$ & $\begin{array}{l}\text { \% } \\
\text { MAR } \\
(n=22)\end{array}$ & $\begin{array}{l}\text { Antibiotic resistance } \\
\text { patterns }\end{array}$ & MAR Index \\
\hline 1 & 1 & 3 & 13.6 & $\begin{array}{l}\text { AU } \\
\text { AU } \\
\text { AU }\end{array}$ & 0.003 \\
\hline 2 & 2 & 1 & 4.5 & $\mathrm{AM} / \mathrm{AU}$ & 0.007 \\
\hline 3 & 9 & 7 & 31.8 & $\begin{array}{l}\text { S/AM/AU } \\
\text { SXT/SP/AU } \\
\text { OFX/CPX/AU } \\
\text { SP/AM/AU } \\
\text { OFX/AM/AU } \\
\text { SXT/CH/AU } \\
\text { OFX/AM/AU }\end{array}$ & 0.029 \\
\hline 4 & 8 & 8 & 36.4 & $\begin{array}{l}\mathrm{OFX} / \mathrm{S} / \mathrm{AM} / \mathrm{AU} \\
\mathrm{CH} / \mathrm{SP} / \mathrm{AM} / \mathrm{AU} \\
\mathrm{S} / \mathrm{SXT} / \mathrm{CH} / \mathrm{AU} \\
\mathrm{OFX} / \mathrm{SP} / \mathrm{CPX} / \mathrm{AU} \\
\mathrm{OFX} / \mathrm{SXT} / \mathrm{AM} / \mathrm{AU} \\
\mathrm{SXT} / \mathrm{CH} / \mathrm{AM} / \mathrm{AU} \\
\mathrm{SXT} / \mathrm{CH} / \mathrm{CPX} / \mathrm{AU} \\
\mathrm{SXT} / \mathrm{SP} / \mathrm{AM} / \mathrm{AU}\end{array}$ & 0.026 \\
\hline 5 & 9 & 3 & 13.6 & $\begin{array}{l}\mathrm{S} / \mathrm{CH} / \mathrm{CPX} / \mathrm{AU} / \mathrm{CN} \\
\mathrm{SXT} / \mathrm{SP} / \mathrm{CPX} / \mathrm{AM} / \mathrm{AU} \\
\text { S/SXT/CH/AM/AU }\end{array}$ & 0.029 \\
\hline
\end{tabular}

Pefloxacin $=$ PEF, Tarivid=OFX, Septrin $=\mathrm{SXT}$, Gentamycin=CN, Amoxacillin=AM, Ciprofloxacin $=\mathrm{CPX}$, Ampiclox $=\mathrm{APX}$, Chloramphenicol $=\mathrm{CH}$, Zinnacef $=\mathrm{Z}$, Sparflox$\operatorname{acin}=$ Z, Rocephin $=$ R, Augumentin $=$ AU, Erythromycin $=E$, Streptomycin $=S$.

Table 8. Antibiotic Resistance Pattern of Klebsiella pneumoniae from Outdoor Market Air in Ado Ekiti, Ekiti State

\begin{tabular}{|c|c|c|c|c|c|}
\hline $\begin{array}{l}\mathbf{S} / \\
\mathbf{N}\end{array}$ & $\begin{array}{l}\text { No of } \\
\text { Antibi- } \\
\text { otic }\end{array}$ & $\begin{array}{l}\text { Fre- } \\
\text { quency }\end{array}$ & $\begin{array}{l}\% \\
\text { MAR } \\
(n=13)\end{array}$ & Antibiotic resistance patterns & $\begin{array}{l}\text { MAR In- } \\
\text { dex }\end{array}$ \\
\hline 1 & 2 & 1 & 7.7 & PEF/AM & 0.011 \\
\hline 2 & 8 & 5 & 38.5 & $\begin{array}{l}\mathrm{PEF} / \mathrm{SP} / \mathrm{AM} \\
\mathrm{PEF} / \mathrm{CPX} / \mathrm{CN} \\
\mathrm{PEF} / \mathrm{S} / \mathrm{AU} \\
\mathrm{PEF} / \mathrm{SP} / \mathrm{CPX} \\
\mathrm{OFX} / \mathrm{AM} / \mathrm{CN}\end{array}$ & \\
\hline 3 & 6 & 2 & 15.4 & $\begin{array}{l}\mathrm{PEF} / \mathrm{OFX} / \mathrm{AU} / \mathrm{CN} \\
\mathrm{PEF} / \mathrm{S} / \mathrm{AM} / \mathrm{CN}\end{array}$ & 0.032 \\
\hline 4 & 8 & 4 & 30.8 & $\begin{array}{l}\mathrm{PEF} / \mathrm{OFX} / \mathrm{S} / \mathrm{SP} / \mathrm{AU} \\
\mathrm{PEF} / \mathrm{OFX} / \mathrm{CPX} / \mathrm{AU} / \mathrm{CN} \\
\mathrm{PEF} / \mathrm{OFX} / \mathrm{AM} / \mathrm{AU} / \mathrm{CN} \\
\mathrm{PEF} / \mathrm{OFX} / \mathrm{SP} / \mathrm{CPX} / \mathrm{CN}\end{array}$ & 0.044 \\
\hline 5 & 8 & 1 & 7.7 & $\mathrm{PEF} / \mathrm{OFX} / \mathrm{S} / \mathrm{SP} / \mathrm{CPX} / \mathrm{AM} / \mathrm{AU} / \mathrm{CN}$ & 0.044 \\
\hline
\end{tabular}

Pefloxacin $=$ PEF, Tarivid $=$ OFX, Septrin $=$ SXT, Gentamycin $=\mathrm{CN}$, Amoxacillin $=$ AM, Ciprofloxacin $=\mathrm{CPX}$, Ampiclox =APX, Chloramphenicol $=\mathrm{CH}$, Zinnacef $=\mathrm{Z}$, Sparfloxacin $=\mathrm{Z}$, Rocephin $=\mathrm{R}, \mathrm{Au}-$ gumentin $=$ AU, Erythromycin $=E$, Streptomycin $=$ S. 
Table 5. Multiple Antibiotic Resistance Pattern of Enterococcus spp from Outdoor Market Air in Ado Ekiti, Ekiti State

\begin{tabular}{|c|c|c|c|c|c|}
\hline $\mathbf{S} / \mathbf{N}$ & $\begin{array}{l}\text { No of Antibi- } \\
\text { otic }\end{array}$ & Frequency & $\begin{array}{l}\% \text { MAR } \\
(n=20)\end{array}$ & $\begin{array}{l}\text { Antibiotic resistance } \\
\text { patterns }\end{array}$ & MAR Index \\
\hline 1 & 3 & 3 & 15 & $\begin{array}{l}\text { AM } \\
\text { PEF } \\
\text { APX }\end{array}$ & 0.011 \\
\hline 2 & 5 & 4 & 20 & $\begin{array}{l}\mathrm{CPX} / \mathrm{SXT} \\
\mathrm{PEF} / \mathrm{APX} \\
\mathrm{APX} / \mathrm{R} \\
\mathrm{APX} / \mathrm{R}\end{array}$ & 0.017 \\
\hline 3 & 6 & 3 & 15 & $\begin{array}{l}\mathrm{CN} / \mathrm{CPX} / \mathrm{SXT} \\
\mathrm{PEF} / \mathrm{CPX} / \mathrm{S} \\
\mathrm{APX} / \mathrm{CPX} / \mathrm{SXT}\end{array}$ & 0.021 \\
\hline 4 & 8 & 6 & 30 & $\begin{array}{l}\mathrm{PEF} / \mathrm{R} / \mathrm{CPX} / \mathrm{E} \\
\mathrm{APX} / \mathrm{Z} / \mathrm{CPX} / \mathrm{S} \\
\mathrm{PEF} / \mathrm{Z} / \mathrm{R} / \mathrm{SXT} \\
\mathrm{PEF} / \mathrm{R} / \mathrm{CPX} / \mathrm{E} \\
\mathrm{PEF} / \mathrm{APX} / \mathrm{R} / \mathrm{S} \\
\mathrm{CPX} / \mathrm{S} / \mathrm{SXT} / \mathrm{E}\end{array}$ & 0.029 \\
\hline 5 & 9 & 2 & 10 & $\begin{array}{l}\mathrm{APX} / \mathrm{CPX} / \mathrm{S} / \mathrm{SXT} / \mathrm{E} \\
\mathrm{PEF} / \mathrm{APX} / \mathrm{R} / \mathrm{CPX} / \mathrm{E}\end{array}$ & 0.032 \\
\hline 6 & 7 & 1 & 10 & $\begin{array}{l}\mathrm{PEF} / \mathrm{APX} / \mathrm{Z} / \mathrm{R} / \mathrm{CPX} / \mathrm{S} \\
\mathrm{XT} / \mathrm{E}\end{array}$ & 0.025 \\
\hline
\end{tabular}

Pefloxacin $=$ PEF, Tarivid $=$ OFX, Septrin $=$ SXT, Gentamycin $=C N$, Amoxacillin $=A M$, Ciprofloxacin $=\mathrm{CPX}$, Ampiclox $=$ APX, Chloramphenicol $=\mathrm{CH}$, Zinnacef $=\mathrm{Z}$, Sparfloxacin $=\mathrm{Z}$, Rocephin $=\mathrm{R}, \mathrm{Au}-$ gumentin $=\mathrm{AU}$, Erythromycin $=\mathrm{E}$, Streptomycin $=\mathrm{S}$.

\section{DISCUSSION}

The outdoor markets are full of different activities that generate dust and release diverse microorganisms into the air. Bacteria are ubiquitous in the atmosphere with the concentration of bacteria cells typically exceeding 100 million $\mathrm{m}^{3}$. They are found in the human populated environment and seen to cause various infectious diseases in both the young and old. In this study, Staphylococcus aureus (17), Escherichia coli (19), Salmonella typhimurium (11), Enterococcus spp (20), Pseudomonas aeruginosa (22) and Klebsiella pneumoniae (22), were recovered. Staphylococcus aureus is a normally part of the skin flora. About $20 \%$ of the human populations are long term carriers of $S$. aureus. $S$. aureus are known to form aggregates in nature, so they tend to yield the higher colony counts, also because of the possible breaking up of the clusters (5). This bacterium is known to be carried in the nasopharynx, throat, skin, cuts, boils, nails and as such, it can easily contribute to the microflora of these environments where the high human population activities occur (6). E. coli is a Gram negative, facultatively anaerobic rod shaped bacterium that is an indicator of recent faecal contamination. Most E. coli strains are harmless but some serotypes can cause a serious food poisoning in humans and are occasionally responsible for the contamination of food products (7). According to Shoemaker and Singh, E. coli strains were isolated from settled dust in the indoor environments (8).
Klebsiellae are major pathogens in the healthcare associated infections (HAI). This is not only because of the significant morbidity associated with the infections but also because the increasing rates of resistance in them make it more difficult for them to be treated with cheaper first line antibiotics. This is related to the work done by Al-Mijalli (9) where S. aureus has a high population distribution than the other organisms isolated due to the fact that it is a normal microflora of humans. Pseudomonas are known to survive in the variety of environmental niche. Pseudomonas aeruginosa can persist on the inanimate objects for 6 hours -16 weeks, and up to 5 weeks on dry surfaces (10). The principal reservoirs for Pseudomonas species are hands of the personnel, sinks or other moist surfaces, transmission is known to occur primarily via hands. However, the airborne dissemination has been implicated to play a significant role in patient-to-patient spread (11). Enterococci are Gram positive, non-spore-forming, obligately fermentative chemo organotrophs. They are catalase negative, although some species produce pseudocatalase, and they are usually homo fermentative, producing the lactic acid (12). In general, enterococci are commensal bacteria, potentially helping in digestion and other gut metabolic pathways. Some Enterococci spp., such as E. faecium and E. faecalis, are used in probiotics to treat diarrhea and improve the host immunity (13). While most species of Enterococci are commensal organisms, some species are opportunistic human pathogens. E. faecalis and E. faecium have become 
particularly important etiological agents of nosocomial infections (14), including urinary tract infections, endocarditis, bacteremia, neonatal infections, central nervous system (CNS) infections, and abdominal and pelvic infections (15).

Therefore, the health risk which these organisms can cause in the human populated environment is enormous. One of the conditions that lead to the prevalence and survival of the organisms isolated from the outdoor environment is their ability to withstand the ultra violet radiation from the sunlight which increases their resistance factor to the antibiotics administered. Bacterial disease has been a perennial problem in the human population since the beginning of the antibiotic era. The widespread use of antibiotics is thought to have engendered evolutionary changes in bacteria that allow them to survive powerful antibiotics (16). The relationship between the antibiotic use and resistance is complex; the major driving factor for the antibiotic resistance is antibiotic use/abuse both in medicine and veterinary medicine. In Nigeria, 53\% of correspondents in a survey took incomplete regimen of antibiotics, a significant proportion of which were self-prescribed for unspecified ailments (17). The antibiotic resistance may also occur by the lowering of the antibiotic concentration which is effective by introducing more mutations that support bacterial growth in the higher antibiotic concentration (18). The antibiotic susceptibility result of this research confirmed high resistance of the isolates to commonly used antibiotics. Septrin was the most effective antibiotic against $S$. typhimurium and $K$. aeruginosa and also Amoxacillin was the most effective for Enterococcus sp. Erythromycin was the most effective against $S$. aureus. Tarivid was the most effective against $E$. coli. Pefloxacin was the most effective against $P$. aeruginosa. Mulu et al. (2006) (19) stated that Gentamicin, Norfloxacin, Ciprofloxacin, Vancomycin and Amikacin were the most effective antibiotics for all bacterial isolates. The antibiotic resistance reported in this study showed that resistance occurs in all bacteria isolates, this report is similar to the one done by Fithamlak et al., 2017 (20)where the antibiotic resistance occurs in all bacterial isolates.

Multiple antibiotic resistance (MAR) is a phenomenon in which an isolate developed resistance to more than one antibiotic. In this research work, it was observed that a great percentage of the bacteria isolated were resistant to two or more antibiotics used, majorly Pefloxacin, Ciprofloxacin, Gentamycin, Amoxacillin and Augmentin. This may be caused by a lot of factors which include the release of antibiotic waste product into the environment by pharmaceutical industries (21). The use of pesticides and fertilizers by farmers, since the farm produce also ended up in the market for sales and bacteria isolates have a greater chance of developing resistance. The abuse of antibiotics which includes misused and self-medication. The antibiotic resistance leads to higher medical costs, prolonged hospital stays, and increased mortality.

\section{CONCLUSION}

This study reveals that most bacteria cells present in the outdoor market environment are mostly bacteria that are the leading cause of various types of infection and allergic reactions in the human populated environment. The resistance of these bacteria to commonly used antibiotics is of enormous clinical and public health implications.

\section{LITERATURE}

1. Naz N, Nasim FU H, Pasha TS. Prevalence of Antibiotic-Resistant Airborne Bacteria along Roadsides in Rahim Yar Khan, Pakistan. Polish. Journal of Environmental Studies 2019; 28(3):1295-1303. doi:10.15244/pj oes/87101.

2. Burrows SM., Albert W., Lawrence M.G., Poschl U. Bacteria in the global atmosphere - Part 1: Review and synthesis of literature data for different ecosystems. Atmosspheric Chemistry and Physics 2009; 9, 9263.

3. Pasquarella C, Pitzurra O, Savino A. The Index of Microbial Air Contamination. The Journal of hospital infection 2001; 46. 241-56. 10.1053/jhin.2000.0820.

4. Krumperman, PH. Multiple antibiotic resistance indexing of Escherichia coli to identify high risk sources of faecal contamination of food. Applied and Environmental Microbiology 1983; 46: 165-170.

5. Lansing AE, Ivnik RJ, Cullum, CM, Randolf C. An empirically derived short form of the Boston naming test. Archives of Clinical Neuropsychology 1999; 14: 481487

6. Jensen SO, Lyon BR Genetics of antimicrobial resistance in Staphylococcus aureus. Future Microbiology 2009; 4(5): 565-582.

7. Kulkarni DS. Preliminary Study of Airborn Microbiota in Some Areas of Amaravati City. Research Journal of Life Sciences, Bioinformatics, Pharmaceutical and Chemical Sciences 2017; 6 (13): 46-50.

8. Shoemaker NB, Wang GR, Salyers AA. Evidence for natural transfer of a tetracycline resistance gene between bacteria from the human colon and bacteria from the bovine rumen. Applied Environmental Microbiology 1992; 58: 1313-1320.

9. Al-mijalli S. Bacterial Contamination of Indoor Air in Schools of Riyadh, Saudi Arabia. Air \& Water Borne Diseases. 2017; 06. 10.4172/2167-7719.1000131.

10. Kramer A, Schwebke I, Kampf G. How long do nosocomial pathogens persist on inanimate surfaces? A systematic review. BMC Infectious Diseases 2006; 6:130130.

11. Panagea S, Winstanley C, Walshaw MJ, Ledson MJ, Hart CA. Environmental contamination with an epidemic strain of Pseudomonas aeruginosa in a Liverpool cystic fibrosis centre, and study of its survival on dry surfaces. Journal of Hospital Infections 2005; 59:102107.

12. Klein, G. Taxonomy, ecology, and antibiotic resistance of enterococci from food and the gastro-intestinal tract. International Journal of Food Microbiology 2003; 88: 123-131. 
13. Franz CM, Huch M, Abriouel H, Holzapfel W, Galvez A. Enterococci as probiotics and their implications in food safety. International Journal of Food Microbiology 2011; 151: 125-140.

14. Morrison, D, Woodford, N. Cookson B. Enterococci as emerging pathogens of humans, p 89S-99S. In Andrew PW, Mitchell TJ (ed), The biology of streptococci and enterococci. Blackwell Science, Oxford, United Kingdom, 1997.

15. Murray BE. (1990). The life and times of the Enterococcus. Clinical Microbiological Review 1990; 3: 46-65.

16. Cooper JE, Feil EJ. The phylogeny of Staphylococcus aureus- which genes make the best intra-species markers. Microbiology 2006; 152: 1297-1305.
17. Yah SC, Yussuf OE, Eghafona NO. Pattern of antibiotic usage by adult populations in the city of Benin, Nigeria. Journal of Public Health Policy 2008; 3: 081-085.

18. Ventola LC. The Antibiotic Resistance Crisis Part 1: Causes and Threats 2015; 40 (4): 277-283.

19. Mulu A, Moges F, Tessem B, Kassu A. Patterns and multiple drug resistance of bacterial pathogens at university of Gondar teaching hospital. Northwest Ethiopia. Ethiopia Medical Journal 2006; 44: 125-131.

20. Fithamlak BS, Fiseha WW, Amsalu AA, Yishak LA. The use of antimicrobials. Annals of clinical Microbiology and Antimicrobials 2017; 16: 29.

21. Joakim-Larsson DG. Antibiotics in the environment. Upsala Journal of Medical Sciences 2014; 119(2):108112. 